\title{
Stripes developed at the strong limit of nematicity in FeSe film
}

\author{
Wei Li ${ }^{1,2,3 \star}$, Yan Zhang ${ }^{2,3,4,5}$, Peng Deng ${ }^{1}$, Zhilin Xu', S.-K. Mo ${ }^{4}$, Ming Yi ${ }^{3}$, Hao Ding ${ }^{1}$, M. Hashimoto ${ }^{6}$, \\ R. G. Moore ${ }^{3}$, D.-H. Lu ${ }^{6}$, Xi Chen ${ }^{1,2 \star}$, Z.-X. Shen ${ }^{3,7 \star}$ and Qi-Kun Xue ${ }^{1,2}$
}

\begin{abstract}
A single monolayer of iron selenide grown on strontium titanate shows an impressive enhancement of superconductivity compared with the bulk', as well as a novel Fermi surface topology ${ }^{2-5}$, extreme two-dimensionality, and the possibility of phonon-enhanced electron pairing ${ }^{1,5}$. For films thicker than one unit cell, however, the electronic structure is markedly different, with a drastically suppressed superconductivity and strong nematicity appearing. The physics driving this extraordinary dichotomy of superconducting behaviour is far from clear. Here, we use low-temperature scanning tunnelling microscopy to study multilayers of iron selenide grown by molecular beam epitaxy, and find a stripe-type charge ordering instability that develops beneath the nematic state. The charge ordering is visible and pinned in the vicinity of impurities. And as it emerges in the strong limit of nematicity, it suggests that a magnetic fluctuation with a rather small wavevector may be competing with the ordinary collinear antiferromagnetic ordering in multilayer films. The existence of stripes in iron-based superconductors, which resemble the stripe order in cuprates, not only suggests that electronic anisotropy and correlation are playing an important role, but also provides a platform for probing the complex interactions between nematicity, charge ordering, magnetism and superconductivity in high-temperature superconductors.
\end{abstract}

Nematicity ${ }^{6}$ in iron-based superconductors, defined as broken rotational symmetry of the tetragonal lattice (the nonequivalence of the $a$ and $b$ axes in Fig. 1a), has been manifested in various studies $^{7-14}$, such as large in-plane anisotropy in neutron scattering ${ }^{7}$ and transport measurements ${ }^{8}$, unidirectional nanostructures in scanning tunnelling microscopy (STM $)^{9}$ and lifting of orbital degeneracy of $d_{x z} / d_{y z}$ bands in angle-resolved photoemission spectroscopy (ARPES) ${ }^{11}$. Although the $d_{x z} / d_{y z}$ band splitting has been observed in both FeSe single crystals ${ }^{15-17}$ (bulk FeSe) and FeSe films grown on $\mathrm{SrTiO}_{3}(\mathrm{FeSe} / \mathrm{STO})^{18}$, the strength of nematicity in FeSe/STO is much larger than that in bulk FeSe reflected in the much higher nematic phase transition temperature in $\mathrm{FeSe} / \mathrm{STO}^{18}$. Consequently, superconductivity does not recover in the multilayer FeSe/STO even with a thickness of 30 unit cells, while bulk FeSe (ref. 19) becomes superconducting around 8 K. No evidence shows that nematicity hinders the emergence of superconductivity in FeSe (bulk FeSe exhibits superconductivity as well as nematicity); conversely, it has been proposed to cause enhancement of electron pairing to a certain extent ${ }^{3}$. Therefore, there might exist another hidden order competing with superconductivity in multilayer FeSe/STO.

FeSe exhibits intriguing magnetic properties as well. For example, although long-range magnetic order is absent in bulk FeSe with rather large local magnetic moment, the commonly found collinear antiferromagnetism emerges rapidly under high pressure ${ }^{20-22}$. This indicates that the carriers of FeSe are in a delicate balance in the spin channel, which may also account for the potential high-temperature electron pairing. Here we combine molecular beam epitaxy (MBE) and STM together to reveal the mystery of FeSe.

Figure $1 \mathrm{~b}$ shows a typical STM topographic image of a 30-unitcell FeSe film grown on STO. The surface is divided into multiple domains with maze-like patterns, which are spread throughout the whole sample surface and could be attributed to the boundaries between two perpendicular nematic domains. Impurities (dark dots in Fig. $1 \mathrm{~b}, \mathrm{c}$ ) are introduced as scattering centres to reveal the electronic interactions in FeSe film. Most of the impurities present a dumbbell-shaped structure $e^{23,24}$ and are probably the Fe vacancies ${ }^{25}$ underneath the Se-terminated surface (Fig. 1c). The boundaries are highlighted by orange dashed lines. An intriguing behaviour is that the stripe pattern could be induced in the vicinity of the impurities. The stripes are unidirectional within each domain, while the orientation of the stripes (marked by white arrows) rotates $90^{\circ}$ when crossing the domain boundary. The existence of the stripes as well as the domains indicates the two-fold symmetry of the underlying electronic structure, and provides direct evidence of the existence of nematicity in FeSe/STO. Hereafter the boundaries will be referred to as $\mathrm{C}_{2}$ domain walls, which are also the fingerprint of nematicity in FeSe films studied by STM. The typical width of the domain wall is $\sim 4 \mathrm{~nm}$ (Supplementary Note 1 ). The high-resolution STM topographic image (inset of Fig. 1c) exhibits the Se-Se lattice as well as the stripes simultaneously, from which the periodicity of $\sim 1.9 \mathrm{~nm}$ and orientation (along the diagonal direction of the $\mathrm{Se}-\mathrm{Se}$ lattice, that is, the direction of the Fe-Fe lattice) of the stripes are determined.

The stripe feature is robust and could be clearly resolved over a rather wide energy range in STM topography. As shown in Fig. 2a-d, stripes are pinned by four dumbbell-shaped impurities, which act as landmarks as well to inspect the details of the stripes. A large number of impurities have been inspected and the induced stripes are all identical; therefore, the phase of the stripes is pinned by the

\footnotetext{
${ }^{1}$ State Key Laboratory of Low-Dimensional Quantum Physics, Department of Physics, Tsinghua University, Beijing 100084, China. ${ }^{2}$ Collaborative Innovation Center of Quantum Matter, Beijing 100084, China. ${ }^{3}$ Stanford Institute for Materials and Energy Sciences, SLAC National Accelerator Laboratory and Stanford University, Menlo Park, California 94025, USA. ${ }^{4}$ Advanced Light Source, Lawrence Berkeley National Lab, Berkeley, California 94720, USA. ${ }^{5}$ International Center for Quantum Materials, School of Physics, Peking University, Beijing 100871, China. ${ }^{6}$ Stanford Synchrotron Radiation Lightsource, SLAC National Accelerator Laboratory, Menlo Park, California 94025, USA. ${ }^{7}$ Departments of Physics and Applied Physics, and Geballe Laboratory for Advanced Materials, Stanford University, Stanford, California 94305, USA. *e-mail: weili83@tsinghua.edu.cn; xc@mail.tsinghua.edu.cn; zxshen@stanford.edu
} 
a

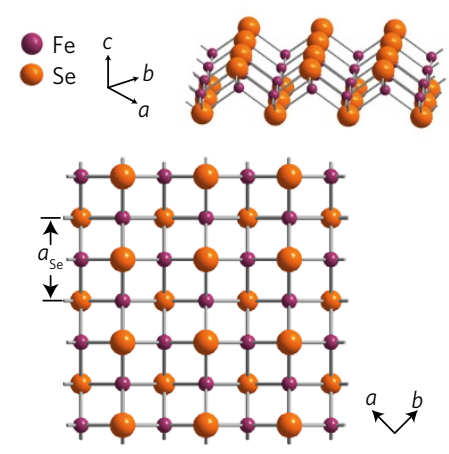

b

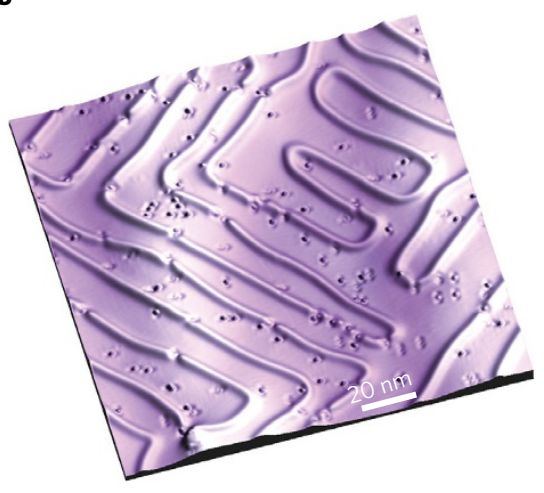

c

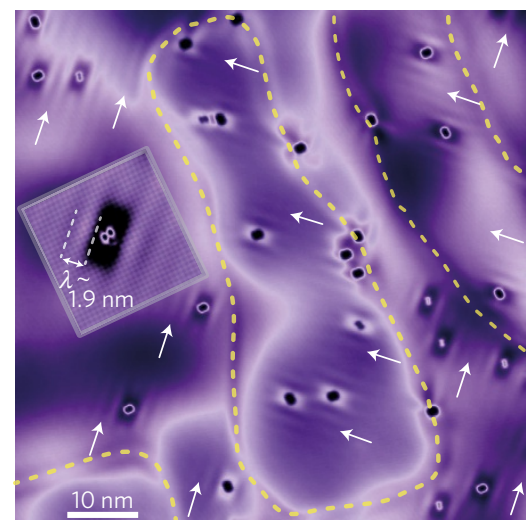

Figure 1| MBE film and $\mathbf{C}_{2}$ domains of FeSe. a, Crystal structure of FeSe. $\mathbf{b}$, STM topographic image of a FeSe film $(150 \mathrm{~nm} \times 150 \mathrm{~nm}$, bias voltage $V=-50 \mathrm{mV}$, tunnelling current $l_{\mathrm{t}}=100 \mathrm{pA}$ ). The maze-like patterns are $C_{2}$ domain walls between two neighbouring nematic domains perpendicular to each other. c, Stripes induced by impurities $(70 \mathrm{~nm} \times 70 \mathrm{~nm}, 60 \mathrm{mV}, 10 \mathrm{pA}$ ). The stripes are of two-fold symmetry (marked by the white arrows), and rotated $90^{\circ}$ when crossing the domain walls (the yellow dashed lines). From the inset ( $10 \mathrm{~nm} \times 10 \mathrm{~nm}, 60 \mathrm{mV}, 200 \mathrm{pA}$ ), the periodicity $\sim 1.9 \mathrm{~nm}$ and orientation of the stripes can be determined. The apparent heights and contrasts of domain walls in $\mathbf{b}$ and $\mathbf{c}$ are different due to the different bias voltages applied (Supplementary Note 1). The periodicity of the stripes can also be extracted by fast Fourier transform of the real space images (Supplementary Note 2).

a

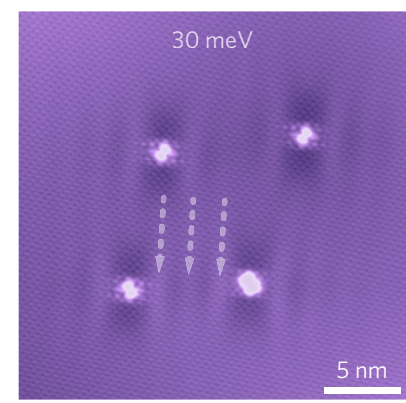

b

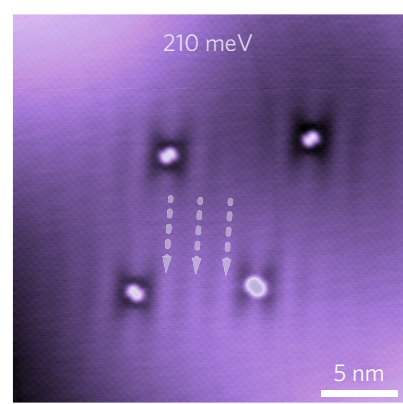

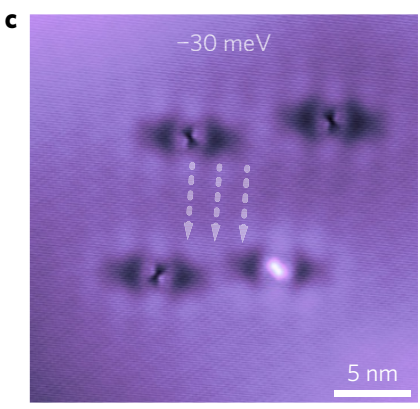

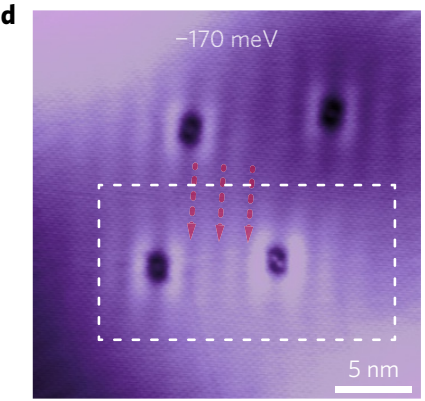

Figure $\mathbf{2}$ | Bias voltage dependence of the stripes in the vicinity of the impurities. a-d, STM topography of the stripes induced by four dumbbell-shaped impurities obtained with different bias voltages. The arrows highlight the stripes between two impurities and the colour of the arrow denotes the stripes' phase change of $180^{\circ}$. The scan size of all images here is $25 \mathrm{~nm} \times 25 \mathrm{~nm}$ and the tunnelling current is $100 \mathrm{pA}$.

impurities. The arrows in Fig. 2 indicate the stripes between two impurities. With various bias voltages, the periodicity of the stripes is unchanged (static), while its phase changes by $180^{\circ}$ under $-30 \mathrm{mV}$ (denoted by colours of the arrows). The above behaviour of the stripes is reminiscent of charge ordering in charge density wave materials. An alternative explanation for the stripes is quasiparticle interference (QPI) induced by impurities, which reflects the band structure information of the system. However, it is unlikely because of the following reasons. The STM topographic image represents the convolution of the density of states within an energy range from the Fermi level $\left(E_{\mathrm{F}}\right)$ to the sampled bias voltage, and thus usually smears out any QPI feature in real space due to drastic variation of energydependent scattering wavevectors (especially at high bias voltages).

The charge ordering (CO) origin of the stripes is further supported by $\mathrm{d} I / \mathrm{d} V$ maps as shown in Fig. 3. We focus on the area within the white dashed line in Fig. 2 d, where two impurities as well as the induced stripe patterns are included. The $\mathrm{d} I / \mathrm{d} V$ map shows the spatial distribution of the density of states at a specific energy, from which the scattering wavevectors can be extracted. Sweeping the bias voltage $V$ (energy), a series of energydependent wavevectors are mapped out. This is the basic idea of the QPI method, and the obtained wavevectors usually arise from the inter- or intra-band scattering of quasiparticles. The most striking feature in the $\mathrm{d} I / \mathrm{d} V$ maps (Fig. 3) is the static (non-dispersive) stripe pattern persisting within an extremely large energy range from $-200 \mathrm{meV}$ to $500 \mathrm{meV}$. This is decisive evidence of CO rather than QPI origin of the stripes, since no band near $E_{\mathrm{F}}$ has such a large band width in FeSe. Meanwhile, the contribution of intra-band scattering (QPI) near $E_{\mathrm{F}}$ can also be observed (highlighted by orange arcs) in the $\mathrm{d} I / \mathrm{d} V$ maps (Fig. $3 \mathrm{i}-\mathrm{m}$ ), corresponding to a holelike band (see the band structure and wavevector marked by green arrows in Supplementary Fig. 4) near $E_{\mathrm{F}}$. The unidirectional QPI features at negative bias voltages are perpendicular to the orientation of the stripes. On the basis of that, we determine that the orientation of the stripes is along the $a$ axis (Supplementary Notes 2 and 3).

We now turn to discuss the driving force of the $\mathrm{CO}$ and its connection with nematicity. Figure $4 \mathrm{a}$ shows $\mathrm{C}_{2}$ domain walls at $77 \mathrm{~K}$, indicating the persistence of nematicity at this temperature. The $\mathrm{C}_{2}$ domain walls gradually disappear at elevated temperatures (Fig. $4 \mathrm{~b}-\mathrm{d}$ ) and the nematic transition $\left(T^{*}\right)$ happens around $120 \mathrm{~K}$, consistent with our previous ARPES measurement ${ }^{18}$. However, the stripes are absent at $77 \mathrm{~K}$ (see the zoom-in topography in Fig. 4e), indicating a new temperature scale for the development of $\mathrm{CO}$. Temperature-dependent measurement of the stripes (Fig. 4e-h) demonstrates that the transition temperature of $\mathrm{CO}\left(T_{\mathrm{CO}}\right)$ is around $60 \mathrm{~K}$. More importantly, the periodicity of the stripes is not sensitive to temperature once formed (see the highlighted area marked by white arrows and red dashed lines in Fig. $4 \mathrm{f}-\mathrm{h}$ at $60 \mathrm{~K}, 45 \mathrm{~K}$ and $4 \mathrm{~K}$, respectively).

Figure $4 \mathrm{i}$ is a phase diagram summarized from the temperaturedependent measurements of the stripes and nematicity. The nematicity appears at $120 \mathrm{~K}$ and is gradually enhanced at lower temperature, and then the $\mathrm{CO}$ develops around $60 \mathrm{~K}$. Given the facts that the stripes emerge within the nematic phase and the stripes are 
$\mathbf{a}$

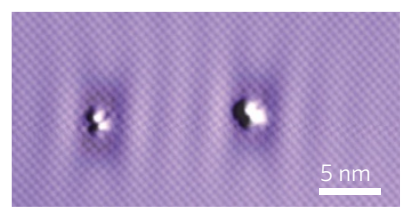

b
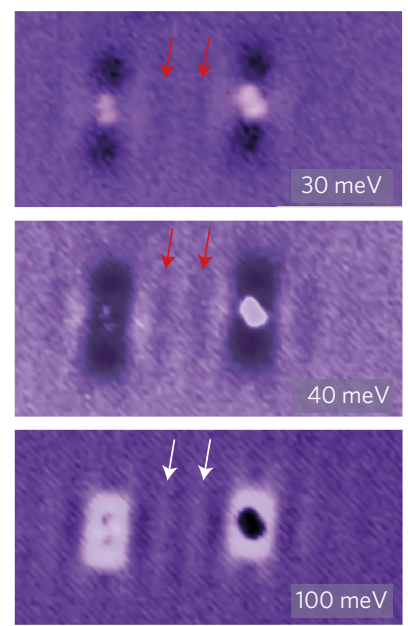
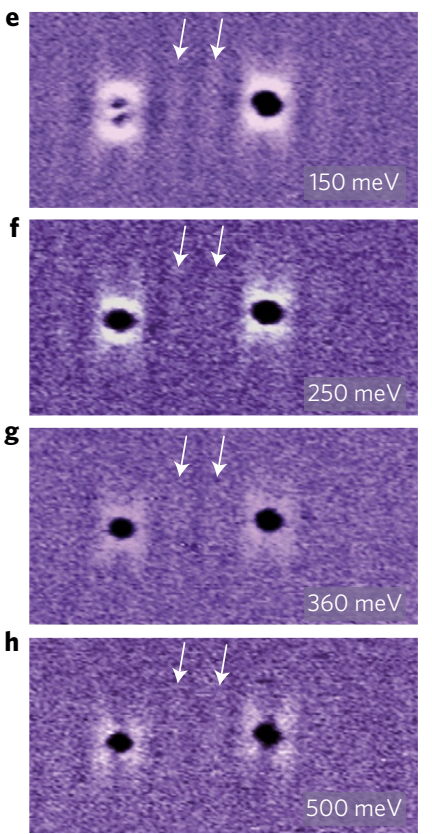
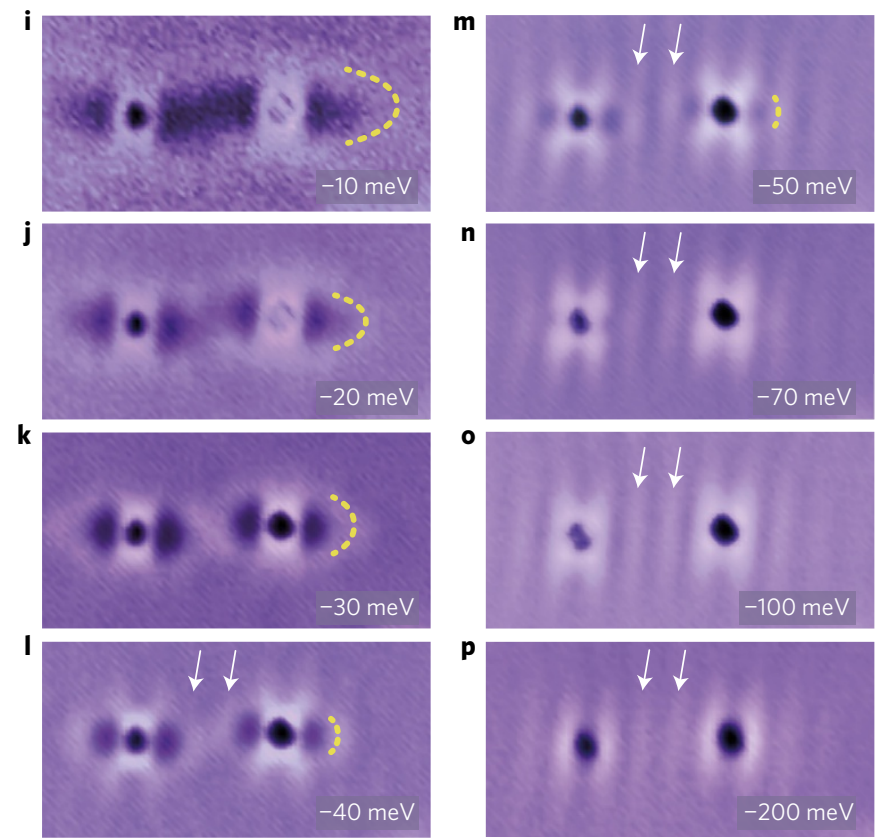
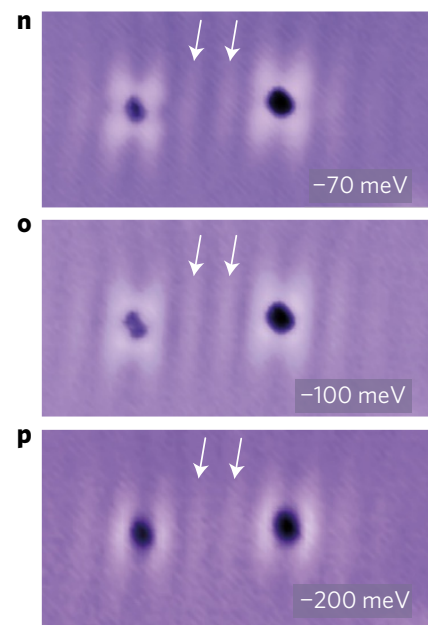

Figure $\mathbf{3}$ | Charge ordering origin of the stripes. a, STM topography of the stripes near two impurities ( $20 \mathrm{~nm} \times 10 \mathrm{~nm}, 60 \mathrm{mV}, 200 \mathrm{pA}$ ), which is the area within the white dashed line in Fig. 2d. b-p. Spatially resolved density-of-state maps at different energies obtained on the same area as $\mathbf{a}$. Set point for $\mathbf{b}$-e and $\mathbf{i}-\mathbf{p}: V=200 \mathrm{mV}, l_{\mathrm{t}}=400 \mathrm{pA}$; set points for $\mathbf{f}, \mathbf{g}: \mathrm{V}=500 \mathrm{mV}, \mathrm{l}_{\mathrm{t}}=500 \mathrm{pA}$. The white and red arrows indicate the static stripes and the orange dashed curves highlight the quasiparticle interferences from low-energy dispersive bands.
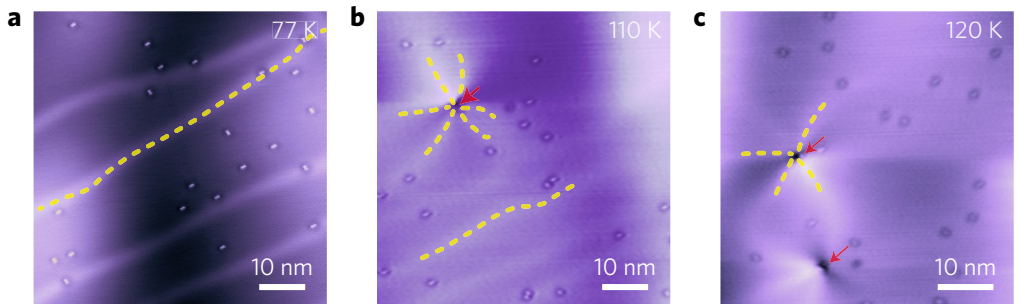

e
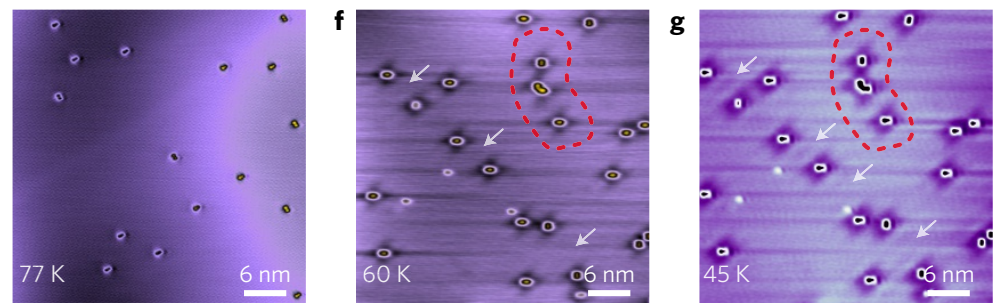
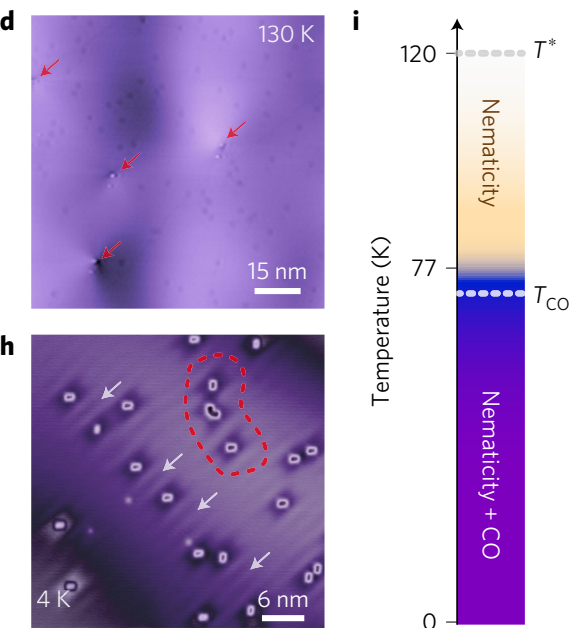

Figure 4 | Temperature dependence of the stripes and the $C_{2}$ domain walls. a-d, The $C_{2}$ domain wall (the yellow dashed lines) gradually disappears at elevated temperatures. $C_{2}$ domain walls could only be pinned near the defects as marked by the red arrows in $\mathbf{c}$ at $120 \mathrm{~K}$ and completely disappear at $130 \mathrm{~K}$ in d. Set point: $V=-60 \mathrm{mV}, I_{\mathrm{t}}=6 \mathrm{pA}$. e-h, Stripes at varied temperatures. The stripes are absent at $77 \mathrm{~K}$ in $\mathbf{e}$. In $\mathbf{f}-\mathbf{h}$, the stripes are clearly visible and the periodicity is independent of temperature from $60 \mathrm{~K}$ to $4 \mathrm{~K}$. Set point for $\mathbf{e}-\mathbf{h}: V=60 \mathrm{mV}, \mathrm{l}_{\mathrm{t}}=6 \mathrm{pA}$. $\mathbf{i}$, Phase diagram based on temperature-dependent measurements of the stripes.

absent in bulk FeSe that exhibits weaker nematicity ${ }^{17}$, the strength of nematicity hence is crucial to the formation of the stripes. An instinctive picture to understand the nematicity-induced CO is: the bandwidth of $d_{x z}$ band becomes wider while the $d_{y z}$ band becomes narrower (Supplementary Fig. 4) with larger anisotropy (nematicity $)^{18}$, and then the electrons tend to be more itinerant along one direction but more localized (to stripe) along the perpendicular one. A straightforward explanation of the $\mathrm{CO}$ is the Fermi surface nesting picture. However, it is unlikely due to the temperature independence of the stripes. At elevated temperatures, the strength of the nematicity as well as the bands splitting size decrease, giving rise to significant variations of the Fermi surfaces ${ }^{18}$, which cannot contribute a temperature-independent wavevector in momentum space.
Besides nematicity, another essential ingredient to induce the stripes is the impurity, since the $\mathrm{CO}$ is always observed in the vicinity of impurities. The correlation length of the induced stripes is $\sim 2.1 \mathrm{~nm}$ (Supplementary Note 4 ). The dumbbell-shaped impurities are probably the Fe vacancies, commonly found in bulk FeSe (refs 23-25). They have two possible orientations along the Se-Se lattice directions that are perpendicular to each other, representing the absence of one $\mathrm{Fe}$ atom (Fe vacancy) in the $\mathrm{Fe}-\mathrm{Fe}$ lattice (see in Fig. $5 c, d$ ). One role of the iron vacancy is to further break the two-fold symmetry ${ }^{26}$, and the strength of nematicity therefore is enhanced locally, which promotes the emergence of the stripes.

$\mathrm{d} I / \mathrm{d} V$ spectra near the stripes do not show any gap-like features (Supplementary Fig. 6 and Supplementary Note 5), suggesting a 

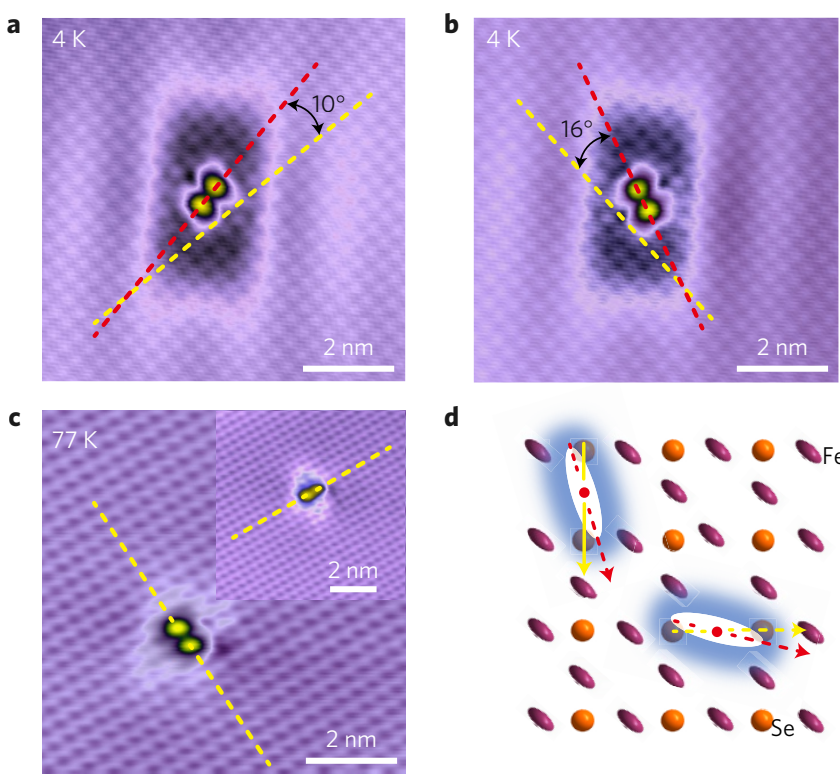

d

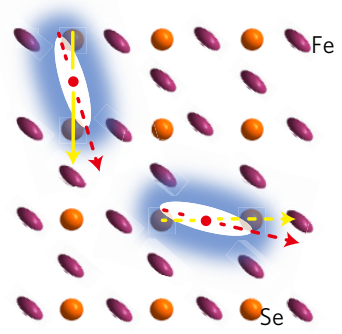

Figure 5 | The interaction between the stripes and the impurity states. $\mathbf{a}, \mathbf{b}$, Distortion of the impurity states due to the interaction with $\mathrm{CO}$. In $\mathbf{a}$ and $\mathbf{b}$, the deflection angle of the 'dumbbell' is larger than $10^{\circ}$ at $4 \mathrm{~K}$. c, Impurity states at $77 \mathrm{~K}$. The deflection angle of the 'dumbbell' is negligible when the $\mathrm{CO}$ is absent. Set points: $V=30 \mathrm{mV}, I_{\mathrm{t}}=6 \mathrm{pA}$. d, Schematic of the distortion at low temperature. The electronic states are elongated near $E_{F}$ due to the large nematicity, and the impurity states tend to align with the stripes.

partial bandgap opening in momentum space. Due to the small amount of impurities and the limited areas with stripes in FeSe film, ARPES that takes the average of the signal from an area of tens of square micrometres could not reveal the band folding and gap opening stemming from the CO. The impurity states (Supplementary Note 5) are strongly distorted by interacting with the CO. As shown in Fig. 5a,b, the directions of the dumbbell shapes (denoted by red dashed lines) show obvious deviation of larger than $10^{\circ}$ from that of the Se-Se lattice (yellow dashed lines). The impurity states interact with the $\mathrm{CO}$ and tend to align with the stripes, giving rise to the observed deviation. At $77 \mathrm{~K}$, with the absence of the stripes, the deviation disappears and the two impurities are perpendicular to each other again (Fig. 5c).

The appearance of stripes near the Fe vacancy indicates a possible magnetic fluctuation in the Fe plane. The impurities help to pin the fluctuation, which is then captured by the STM tip in the charge channel. Long-range magnetic order is absent but the collinear AFM emerges rapidly under high pressure in bulk FeSe (refs 20-22), indicating that there might be two comparable competing orders in the spin channel at ambient pressure. A tiny change of the lattice constant may break the balance. Positive pressure to FeSe causes emergence of collinear $\mathrm{AFM}^{20-22}$. Conversely, tensile stress is applied when FeSe is grown on STO, equivalent to applying a negative pressure to the system. Therefore, the other side of the pressure-related phase diagram of FeSe is reached, within which the correlation of the whole system is enhanced and the other magnetic order wins. A magnetic fluctuation with a rather small $q \sim \pi / 5$ is predicted in bulk FeSe (ref. 27), quantitatively comparable to the periodicity of the observed stripes. We therefore attribute the observed stripes to the origin of a charge ordering induced by the magnetic fluctuation. The two possible fluctuations in the spin channel might be crucial to understand the unique properties of FeSe, such as the magnetism under pressure ${ }^{20-22}$ and electron pairing at rather high temperatures ${ }^{1,20}$.

The existence of $\mathrm{CO}$ instability also provides a natural explanation for the suppression of superconductivity in multilayer
FeSe/STO. As commonly occurs in other materials, such as cuprates and transition metal dichalcogenides, superconductivity always competes with $\mathrm{CO}$. Besides the impurities we discussed above, the widespread $\mathrm{C}_{2}$ domain walls induce the stripes as well where the domain walls are aligned with the intrinsic stripe directions (Supplementary Fig. 7). This could play a more important role to break the long-range coherence of Cooper pairs in multilayer FeSe films.

The stripe-type charge ordering in iron-based superconductor resembles that in cuprates and suggests the importance of electronic anisotropy and correlation. Our findings shed new light on understanding the complex relationship between nematicity, charge ordering, magnetism and superconductivity in hightemperature superconductors.

\section{Methods}

Methods, including statements of data availability and any associated accession codes and references, are available in the online version of this paper.

Received 25 February 2017; accepted 23 May 2017; published online 17 July 2017

\section{References}

1. Wang, Q. Y. et al. Interface induced high temperature superconductivity in single unit-cell FeSe films on $\mathrm{SrTiO}_{3}$. Chin. Phys. Lett. 29, 037402 (2012).

2. Liu, D. F. et al. Electronic origin of high-temperature superconductivity in single-layer FeSe superconductor. Nat. Commun. 3, 931 (2012).

3. Tan, S. Y. et al. Interface-induced superconductivity and strain-dependent spin density waves in $\mathrm{FeSe} / \mathrm{SrTiO}_{3}$ thin films. Nat. Mater. 12, 634-640 (2013).

4. He, S. L. et al. Phase diagram and electronic indication of high-temperature superconductivity at $65 \mathrm{~K}$ in single-layer FeSe films. Nat. Mater. 12, 605-610 (2013).

5. Lee, J. J. et al. Interfacial mode coupling as the origin of the enhancement of $T_{c}$ in FeSe films on $\mathrm{SrTiO}_{3}$. Nature 515, 245-248 (2014).

6. Kivelson, S. A., Fradkin, E. \& Emery, V. J. Electronic liquid-crystal phases of a doped Mott insulator. Nature 393, 550-553 (1998).

7. Zhao, J. et al. Spin waves and magnetic exchange interactions in $\mathrm{CaFe}_{2} \mathrm{As}_{2}$. Nat. Phys. 5, 555-560 (2009)

8. Chu, J. H. et al. In-plane resistivity anisotropy in an underdoped iron arsenide superconductor. Science 329, 824-826 (2010).

9. Chuang, T.-M. et al. Nematic electronic structure in the 'parent' state of the iron-based superconductor $\mathrm{Ca}\left(\mathrm{Fe}_{1-x} \mathrm{Co}_{x}\right)_{2} \mathrm{As}_{2}$. Science 327, 181-184 (2010).

10. Kasahara, S. Electronic nematicity above the structural and superconducting transition in $\mathrm{BaFe}_{2}\left(\mathrm{As}_{1-x} \mathrm{P}_{x}\right)_{2}$. Nature 486, 382-385 (2012).

11. Yi, M. et al. Symmetry-breaking orbital anisotropy observed for detwinned $\mathrm{Ba}\left(\mathrm{Fe}_{1-x} \mathrm{Co}_{x}\right)_{2} \mathrm{As}_{2}$ above the spin density wave transition. Proc. Natl Acad. Sci. USA 108, 6878-6883 (2011).

12. Rosenthal, E. P. et al. Visualization of electron nematicity and unidirectional antiferroic fluctuations at high temperatures in NaFeAs. Nat. Phys. 10, 225-232 (2014).

13. Tanatar, M. A. et al. Uniaxial-strain mechanical detwinning of $\mathrm{CaFe}_{2} \mathrm{As}_{2}$ and $\mathrm{BaFe}_{2} \mathrm{As}_{2}$ crystals: optical and transport study. Phys. Rev. B 81, 184508 (2010).

14. Lee, C.-C., Yin, W. G. \& Ku, W. Ferro-orbital order and strong magnetic anisotropy in the parent compounds of iron-pnictide superconductors. Phys. Rev. Lett. 103, 267001 (2009).

15. Nakayama, K. et al. Reconstruction of band structure induced by electronic nematicity in an FeSe superconductor. Phys. Rev. Lett. 113, 237001 (2014).

16. Borisenko, S. V. et al. Direct observation of spin-orbit coupling in iron-based superconductors. Nat. Phys. 12, 311-317 (2016).

17. Watson, M. D. et al. Evidence for unidirectional nematic bond ordering in FeSe. Phys. Rev. B 94, 201107(R) (2016).

18. Zhang, Y. et al. Distinctive orbital anisotropy observed in the nematic state of a FeSe thin film. Phys. Rev. B 94, 115153 (2016).

19. Hsu, F. C. et al. Superconductivity in the PbO-type structure $\alpha$-FeSe. Proc. Natl Acad. Sci. USA 105, 14262-14264 (2008).

20. Bendele, M. et al. Coexistence of superconductivity and magnetism in $\mathrm{FeSe}_{1-x}$ under pressure. Phys. Rev. B 85, 064517 (2012).

21. Sun, J. P. et al. Dome-shaped magnetic order competing with high-temperature superconductivity at high pressures in FeSe. Nat. Commun. 7, 12146 (2016).

22. Kothapalli, K. et al. Strong cooperative coupling of pressure-induced magnetic order and nematicity in FeSe. Nat. Commun. 7, 12728 (2016).

23. Song, C.-L. et al. Suppression of superconductivity by twin boundaries in FeSe. Phys. Rev. Lett. 109, 137004 (2012).

24. Kasahara, S. et al. Field-induced superconducting phase of FeSe in the BCS-BEC cross-over. Proc. Natl Acad. Sci. USA 111, 16309-16313 (2014). 
25. Huang, D. et al. Dumbbell defects in FeSe films: a scanning tunneling microscopy and first-principles investigation. Nano Lett. 16, 4224 (2016)

26. Inoue, Y., Yamakawa, Y. \& Kontani, H. Impurity-induced electronic nematic state and $\mathrm{C}_{2}$-symmetric nanostructures in iron pnictide superconductors. Phys. Rev. B 85, 224506 (2012).

27. Tam, Y.-T., Yao, D.-X. \& Wei, K. Itinerancy-enhanced quantum fluctuation of magnetic moments in iron-based superconductors. Phys. Rev. Lett. 115, 117001 (2015)

\section{Acknowledgements}

We thank T. Li, H. Yao and J. P. Hu for discussions. STM work was supported by the National Science Foundation (No. 11674191) and Ministry of Science and Technology of China (No. 2016YFA0301002). W.L. was also supported by Beijing Young Talents Plan. ALS and SSRL are operated by the Office of Basic Energy Sciences, US DOE, under Contracts No. DE-AC02-05CH11231 and No. DE-AC02-76SF00515, respectively. The Stanford work is supported by the US DOE, Office of Basic Energy Science, Division of Materials Science and Engineering, under award number DE-AC02-76SF00515.

\section{Author contributions}

W.L., P.D., Z.X. and H.D. carried out the STM experiments; W.L. and Y.Z. performed the ARPES experiments; W.L., X.C. and Z.-X.S. designed and coordinated the experiments; D.-H.L. and M.H. provided experimental support at Stanford Synchrotron Radiation Lightsource. S.K.-M. provided experimental support at Advanced Light Source. Y.Z., D.-H.L., M.Y. and R.G.M. provided discussion about data and interpretation. Q.-K.X. oversaw the project. W.L. wrote the manuscript with comments from all authors.

\section{Additional information}

Supplementary information is available in the online version of the paper. Reprints and permissions information is available online at www.nature.com/reprints. Publisher's note: Springer Nature remains neutral with regard to jurisdictional claims in published maps and institutional affiliations. Correspondence and requests for materials should be addressed to W.L., X.C. or Z.-X.S.

\section{Competing financial interests}

The authors declare no competing financial interests. 


\section{Methods}

FeSe films were grown on Nb-doped (0.05 wt $\%) \mathrm{SrTiO}_{3}(100)$. $\mathrm{TiO}_{2}$-terminated atomically flat surfaces were prepared by degassing at $450{ }^{\circ} \mathrm{C}$ for one hour and subsequent annealing at $1,100^{\circ} \mathrm{C}$ for $20 \mathrm{~min}$. For the FeSe films for STM measurements, high-purity $\mathrm{Fe}$ (99.995\%) and $\mathrm{Se}$ (99.9999\%) were evaporated from two standard Knudsen cells. The growth was carried out under Se-rich conditions with a nominal Se/Fe flux ratio of $\sim 20$. For the FeSe film for ARPES measurements, an electron-beam cell and a thermal cracker effusion cell were used to evaporate $\mathrm{Fe}$ and Se, respectively. The Se/Fe flux ratio was set between 3-4. Substrate temperatures were kept at $370^{\circ} \mathrm{C}$ for both growth methods. The as-grown FeSe films were subsequently annealed at $400^{\circ} \mathrm{C}$ for several hours to remove the excess Se. Both films are of comparable high quality judging from the reflection high-energy electron diffraction (RHEED) patterns, STM and ARPES results.

To introduce iron vacancies as the scattering centres for the STM studies. The as-grown FeSe films were annealed at a lower temperature $\sim 380^{\circ} \mathrm{C}$, and a low density of iron vacancies could be obtained in the films.
In situ STM experiments were conducted in an ultrahigh-vacuum low-temperature (4 K) STM equipped with an MBE chamber for film growth (Unisoku). A polycrystalline PtIr STM tip was used and characterized on a Ag island before STM experiments.

ARPES measurements were performed at the Stanford Synchrotron Radiation Lightsource Beamline (SSRL) 5-4 and the Advanced Light Source (ALS) Beamline 10.1 at $21 \mathrm{~K}$. The as-grown FeSe films were transported to the ARPES chamber for SSRL measurements under ultrahigh vacuum via a vacuum suitcase that is pumped by an ion pump. The FeSe samples measured in ALS were capped with $20 \mathrm{~nm}$ Se as protecting layers. The protecting layers were de-capped by carefully annealing the samples at $400^{\circ} \mathrm{C}$ and monitored by RHEED. The recovery of the bright and sharp RHEED patterns during the heating procedures indicated the clean surface of FeSe films. The samples measured in SSRL and ALS show consistent band structures.

Data availability. The data that support the plots within this paper and other findings of this study are available from the corresponding author on reasonable request. 